\title{
CONCERNING STRONG LIE IDEALS
}

\author{
WILLARD E. BAXTER ${ }^{1}$
}

Let $A$ be a simple ring of characteristic $\neq 2$ or 3 , with either its center $Z=(0)$ or of dimension greater than 16 over its center, and with an involution defined on it. Let $S$ and $K$ be the sets of symmetric and skew elements respectively. The Lie and Jordan products are $[u, v]$ $=u v-v u$ and $u \circ v=u v+v u$. Denote by $[K, K]$ the additive subgroup of $K$ generated by the elements $[k, m], k$ and $m$ in $K$. An additive subgroup, $U$, of $K$ is called a Lie ideal of $K$ if $[u, k]$ is in $U$ for all $u$ in $U, k$ in $K$.

Herstein [2] defines a Lie ideal, $U$, of $K$ to be a strong Lie ideal if $u$ in $U$ implies $u^{3}$ is in $U$. In this paper we investigate the structure of strong Lie ideals in simple rings with involution. The main result of this paper is:

TheOREM 1. If $A$ is as above and $U$ is a strong Lie ideal, then either $U$ is contained in $Z$ or $U=K$.

In order to prove this theorem we make two remarks and prove several lemmas.

REMark 1. $S^{2}=S+[K, K]$. This follows from $S=S \circ S$ (cf. [2, Theorem 8]) and $[S, S]=[K, K]$ (cf. [1, Lemma 10]).

REMARK 2. There exists $k$ in $[K, K]$ such that $k^{2} \notin Z$. This is shown in the proof of Theorem 4 [1].

We use Remark 1 in the proof of the following lemma:

Lemma 1. If $s \in S, s \in Z$ then $K=[K, K]+s \circ[K, K]$ where $s \circ[K, K]$ is the additive subgroup of $K$ generated by $\{s \circ k \mid k \in[K, K]\}$.

Proof. We first note that $s S^{2} \subset s[K, K]+S^{2}$ for all $s \in S$. Let $r, t, s \in S$; then

$$
2 s t r=s(t r-r t)+s(t r+r t) .
$$

Hence the remark follows since the characteristic $\neq 2$.

Now choose $s \in S, s \notin Z$. Then there exists some $r \in S$ such that $s r-r s \neq 0$; otherwise $s \in Z$ (cf. [2, Theorem 9]). Hence, let $t \in S$ so that $s t-t s \neq 0$. Consider $(s t-t s) S^{n}$ where $S^{n}$ is the additive group generated by elements of form $r_{1} \cdots r_{n}, r_{i} \in S$ for all $i$. It follows that

Received by the editors July $18,1959$.

1 The preparation of this paper was supported, in part, by the University of Delaware Research Foundation, Inc. 


$$
(s t-t s) S^{n} \subset S^{2}+s[K, K] .
$$

To see this, consider $(s t-t s) r_{1} \cdots r_{n}$.

$$
\begin{aligned}
(s t-t s) r_{1} \cdots & r_{n} \\
= & s\left[t, r_{1} \cdots r_{n}+r_{n} \cdots r_{1}\right]-t\left(s r_{1} \cdots r_{n}+r_{n} \cdots r_{1} s\right) \\
& +\left(t r_{n} \cdots r_{1} s+s r_{1} \cdots r_{n} t\right)-s\left(t r_{n} \cdots r_{1}+r_{1} \cdots r_{n} t\right) \\
& +s\left(r_{1} \cdots r_{n}+r_{n} \cdots r_{1}\right) t .
\end{aligned}
$$

The latter term is in $s S^{2}$, and thus in $s[K, K]+S^{2}$, and the other terms on the right are in $S^{2}, s[K, K]$ or $S$. Hence we have proved (I).

Since $\bar{S}$, the subring generated by the elements of $S$, is $A$ it follows that $(s t-t s) A \subset S^{2}+s[K, K]$. Also, $[A, A] \subset S^{2}+s[K, K]$ and $S^{2}+s[K, K]=S+[K, K]+s \circ[K, K]$. Thus, for all $a, b \in A$,

$$
b(s t-t s) a \in S+[K, K]+s \circ[K, K],
$$

since $b(s t-t s) a=[b,(s t-t s) a]-(s t-t s) a b$. That is,

$$
A(s t-t s) A \subset S+[K, K]+s \circ[K, K] \text {. }
$$

By assumption $s t-t s \neq 0$ and $A$ is simple; therefore $A=S+[K, K]$ $+s \circ[K, K]$ and we conclude, using the fact that $S \cap K=(0)$, that $K=[K, K]+s \circ[K, K]$ for all $s \in S, s \notin Z$.

We need the following lemma to prove the main theorem:

Lemma 2. Let $U$ be a strong Lie ideal of $K$ such that $U$ contains $[K, K]$. Then if $u$ and $v$ are in $U, 3\left(v^{2} u+u v^{2}\right)$ is in $U$.

Proof. If $u, v \in U$, then $(u+v)^{3},(u-v)^{3}$ and $2 u^{3} \in U$. Hence, $(u+v)^{3}+(u-v)^{3}-2 u^{3} \in U$; that is,

$$
2\left(v^{2} u+v u v+u v^{2}\right) \in U .
$$

Also, $[v,[v, u]] \in U$ and hence their difference, $3\left(v^{2} u+u v^{2}\right)$, is in $U$. . We are now in a position to prove Theorem 1. Herstein [2] has proven that if $A$ is as above and $U$ is a Lie ideal of $K$ then either $U \subset Z$ or $U \supset[K, K]$. In that which follows we assume $U \supset[K, K]$. By Remark 2 there exists $k \in[K, K] \subset U$ such that $k^{2} \in Z$ and hence, by Lemma 2 ,

$$
3\left(k^{2} m+m k^{2}\right) \in U \text { for all } m \in[K, K] .
$$

By assumption, the characteristic $\neq 3$. Thus $[K, K]=3[K, K]$ and

$$
k^{2} n+n k^{2} \in U \text { for all } n \in[K, K] .
$$

However, by Lemma $1, K=k^{2} \circ[K, K]+[K, K]$ and, since $[K, K]$ $\subset U$, it follows that $K=U$. This completes the proof of Theorem 1 . 
It should be noted that Lemma 1 is interesting, independent of strong Lie ideals, for it generalizes a result of [1]. This generalization can be stated as follows:

THEOREM 2. Let $A$ be a simple ring of characteristic $\neq 2$, with either its center $Z=(0)$ or of dimension greater than 16 over its center, and with an involution defined on it; then if either $K, S,[K, K]$ or $[K, S]$ are finite dimensional, $A$ is finite dimensional.

\section{REFERENCES}

1. W. E. Baxter, Lie simplicity of a special class of associative rings. II, Trans. Amer. Math. Soc. vol. 87 (1958) pp. 63-75.

2. I. N. Herstein, Lie and Jordan systems in simple rings with involution, Amer. J. Math. vol. 78 (1956) pp. 629-649.

University of Delaware

\section{TRIANGLE INEQUALITY IN $l$-GROUPS}

\section{J. A. KALMAN}

In $[1$, p. 309] G. Birkhoff remarks that "In a commutative l-group, we can ... prove the triangle inequality $|a+b| \leqq|a|+|b|$, but this does not seem to hold in general." The purpose of this note is to show that in fact if

$$
|a+b| \leqq|a|+|b|
$$

for all $a$ and $b$ in the additive l-group $G$, then $G$ is commutative.

Proof. It is sufficient to show that any two positive elements of $G$ are permutable [2, p. 234]. Suppose therefore that $x$ and $y$ are positive elements of $G$. Taking $a=-x$ and $b=-y$ in (1), we obtain $x+y$ $\geqq|-x-y|=y+x$. Similarly $y+x \geqq x+y$. Hence $x+y=y+x$. This completes the proof.

\section{REFERENCES}

1. G. Birkhoff, Lattice-ordered groups, Ann. of Math. vol. 43 (1942) pp. 298-331.

2. - Lattice theory, Amer. Math. Soc. Colloquium Publications, rev. ed., vol. 25, New York, 1948.

UNIVERSITY OF AUCKLAND

Received by the editors August 1, 1959. 